\title{
Interplay between folding and assembly of fibril-forming polypeptides
}

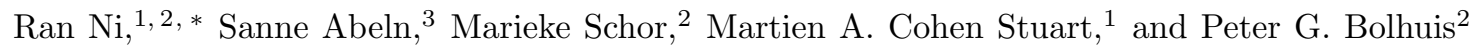 \\ ${ }^{1}$ Laboratory of Physical Chemistry and Colloid Science, \\ Wageningen University, Dreijenplein 6, 6703 HB Wageningen, The Netherlands \\ ${ }^{2}$ Van 't Hoff Institute for Molecular Sciences, Universiteit van Amsterdam, \\ Science Park 904, 1098 XH Amsterdam, The Netherlands \\ ${ }^{3}$ Centre for Integrative Bioinformatics (IBIVU), Vrije Universiteit, \\ De Boelelaan 1081A, 1081 HV Amsterdam, The Netherlands
}

\begin{abstract}
Polypeptides can self-assemble into hierarchically organized fibrils consisting of a stack of individually folded polypeptides driven together by hydrophobic interaction. Using a coarse grained model, we systematically studied this self-assembly as a function of temperature and hydrophobicity of the residues on the outside of the building block. We find the self-assembly can occur via two different pathways - a random aggregation-folding route, and a templated-folding process thus indicating a strong coupling between folding and assembly. The simulation results can explain experimental evidence that assembly through stacking of folded building blocks is rarely observed, at the experimental concentrations. The model thus provides a generic picture of hierarchical fibril formation.
\end{abstract}

PACS numbers: 87.14.em, 87.15.A-, 87.15.hp, 87.15.Zg

While, perhaps surprisingly, most proteins, and even short peptides, share the general ability to form fibrils under appropriate conditions [1 $[3$, the interest in filamentous proteins originates to a large extent from their association with neurodegenerative disorders such as Alzheimers and Parkinsons disease [4]. However, protein fibers also have promising applications in biomaterials [5, 6]. For instance, silk-collagen-like triblock copolymers self-assemble into micrometer long fibrils, which form dilute gels with surprisingly high modulus, serving as promising candidates for novel materials such as artificial tissues [7. Similar to amyloid fibrils, these silk-collagen fibrils consist of stacked cross $\beta$ structures made out of the silk-like block, sterically stabilized by the collagen-like hydrophilic flanks. Contrary to amyloid fibrils, the silk-like building blocks are so-called $\beta$-rolls, consisting of two interconnected parallel $\beta$-sheets with a hydrophobic outside surface, which promotes stacking into long fibers [8. The mechanism of such hierarchical protein fibril formation is still poorly understood. Yet, such understanding is crucial for controlling fibril nucleation and growth. One clue comes from the fact that experimental fibril elongation speeds are more than four orders of magnitude smaller than theoretically predicted by diffusion 9. This suggests that the fibril growth (and nucleation) encounters pronounced free energy barriers, possibly due to significant conformational changes, e.g., folding, of each polypeptide. Hierarchical fibril formation thus involves a combination of both folding and assembly processes. One can therefore imagine (at least) three possible scenarios: (i) an aggregationfolding process, in which from disordered aggregates of unfolded polypeptides a fibril structure nucleates and grows, (ii) a templated-folding process, in which existing fibrils sequester unfolded polypeptides and induce them to fold, or (iii) a folding-docking process, in which polypeptides first fold individually before stacking together into fibrils. Computer simulation can complement experiments and yield mechanistic insight, but due to computational challenges, the folding and assembly processes of fibril-forming polypeptides are usually studied separately [8, 10-16. Thus, the interplay between folding and assembly in the self-assembly of polypeptides is still an open question. Here, we investigate such interplay for a system closely related to the silk-collagen-like triblock copolymer, which is, due to its hierarchical structure, an excellent model system.

While in principle atomistic models can provide molecular insight in the folding and assembly process, such all-atom simulations are prohibitively expensive. Instead we employ a coarse-grained polypeptide model in which each residue occupies a single site on a cubic lattice [17, and all other sites are considered as solvent. For each residue a unit vector indicates the direction of the side chain. This highly efficient model captures two essential features for a correct description of folding: the formation of hydrogen bonds and the directionality of side chains. The total potential energy of the system is given by $E=E_{a a}+E_{\text {solvent }}+E_{h b}+E_{\text {steric }}$, where $E_{a a}$ and $E_{\text {solvent }}$ are a knowledge based interaction potential between amino acids, and between amino acids and solvent, respectively (see Table I). This potential has been shown to prevent artificial aggregation of proteins in their native state. $E_{h b}$ is the potential energy of formed hydrogen bonds, and $E_{\text {steric }}$ represents the steric hindrance between consecutive residues in a polypeptide chain [18. Two amino acids in contact interact only when their side chains are either in parallel or pointing towards each other. Similarly, interaction between a residue and solvent only exists when the side chain points to a solvent site. When a residue is not part of a turn in the backbone, it can adopt a strand state, depending on the side 
TABLE I: Interaction matrix (in reduced units) for the residues in the used sequence [17. Amino acids are denoted by their one-letter code ( $\mathrm{I}=$ isoleucine, $\mathrm{A}=$ alanine, $\mathrm{R}=$ arginine, $\mathrm{E}=$ glutamate) and $w$ denotes the solvent (water). The hydrophobicity of alanine is varied via the A-w interaction $\left(\epsilon_{A, w}\right)$ as indicated.

\begin{tabular}{|c|c|c|c|c|}
\hline 1 & & $\mathrm{R}$ & $\mathrm{E}$ & $\mathrm{w}$ \\
\hline I -0.79 & -0.40 & 0.5 & 0.69 & 0.7 \\
\hline A & -0.34 & 0.49 & 0.77 & $0.01 ; 0.1 ; 0.2 ; 0.4 ; 0.6$ \\
\hline $\mathrm{R}$ & & 0.43 & -0.6 & -0.57 \\
\hline $\mathrm{E}$ & & & 1.02 & -0.78 \\
\hline $\mathrm{w}$ & & & & 0.0 \\
\hline
\end{tabular}

chains. Two residues in contact and both in strand state can form a hydrogen bond with an energy $\epsilon_{h b}=-0.5$ (all interaction potentials in this work are in reduced units), when their side chains are aligned. An energy penalty, $\epsilon_{s}=0.55$, prevents that side chains of consecutive residues point in the same direction, mimicking steric hindrance and restrictions in bond rotation. Due to its small size alanine becomes considerably more hydrophobic in a $\beta$-strand environment 19 . This effect is not captured in the original parameterization of the potential. To compensate for this, and to investigate the influence of the hydrophobicity of the building block's surface, the interaction between alanine $(\mathrm{A})$ and water $(\mathrm{w})$ is varied between 0.01 and 0.6 (see Table I). The model is simulated using lattice Monte Carlo with a classic move set [17.

Lattice models in general are not expected to fold natural sequences. We therefore first need to design a sequence to fold into the desired $\beta$-roll structure. Proper folding of a $\beta$-roll on a cubic lattice demands a palindromic sequence and a slightly different topology compared to its off-lattice counterpart: whereas the experimental $\beta$-roll consists of two interconnected parallel $\beta$-sheets, the lattice $\beta$-roll is comprised of antiparallel sheets. Restricting the silk part to 80 residues to make the calculation tractable, the design procedure results in the optimal sequence $\left(E(A I)_{3} R E(I A)_{3} R\right)_{5}$. This sequence has a repetitive nature comparable to the experimental silk block $\left.\left(E(A G)_{3}\right)\right)_{24}$ [9], but with isoleucine replacing glycine. This is not unrealistic, as glycines in $\beta$-sheets are more hydrophobic than the average glycine [19]. The extra (arginine) residue has been introduced to fit the $\beta$-roll structure on the lattice. In experiments, random aggregation of fibrils is prevented by the presence of hydrophilic flanks 9 . A similar effect has been shown by lattice model simulations 20]. To mimic those flanks we attached 10 glutamate residues to both peptide termini. As shown in Fig. 1 the designed polypeptide indeed folds into a $\beta$-roll structure with a hydrophobic outside surface covered with alanine sidechains.

Whereas polypeptide folding is driven by several types

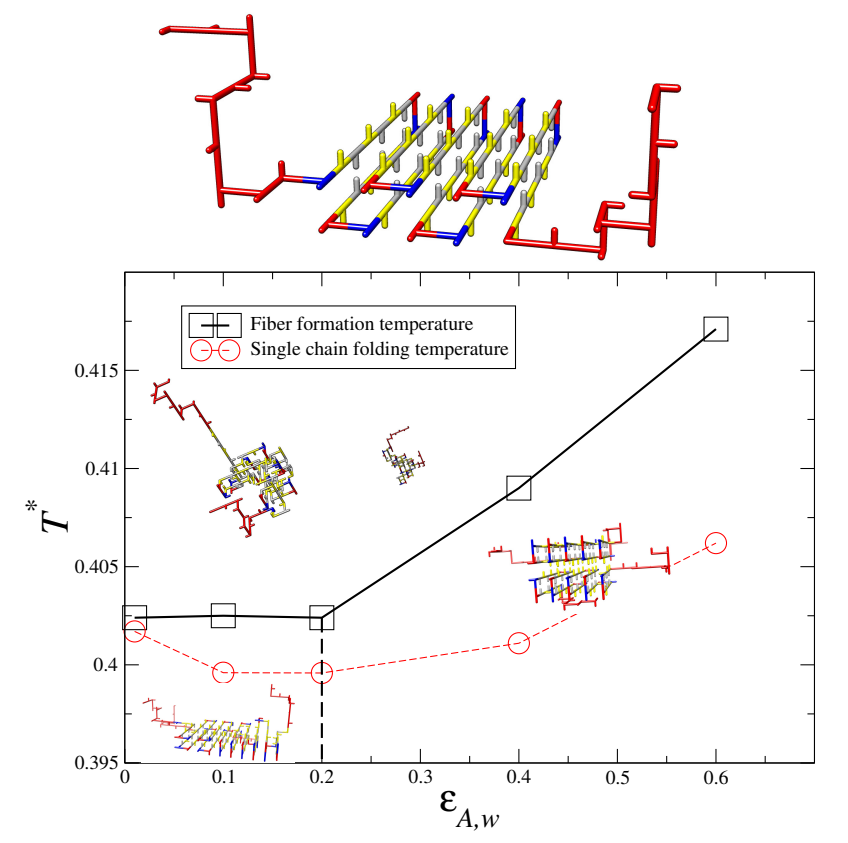

FIG. 1: (color online) Top: Folded $\beta$-roll structure of a single polypeptide $($ alanine $(A)=$ yellow, isoleucine $(\mathrm{I})=$ white, glutamate $(\mathrm{E})=$ red, arginine $(\mathrm{R})=$ blue $)$. Bottom: Equilibrium phase diagram in the $T^{*}-\epsilon_{A, w}$ plane, with transition temperatures for two polypeptides (squares) and the folding temperature of single polypeptide (circles). Typical snapshots of the isolated unfolded, aligned and stacked phases are included.

of interactions, including hydrophobicity and hydrogen bond formation, the assembly is purely hydrophobicity driven 19. To investigate the interplay between folding and assembly, it is necessary to be able to influence both independently. Control parameters in the folding and assembly process are the temperature and the concentration, but these will probably influence both aspects simultaneously. Therefore we also change the hydrophobic strength of the outside surface, by varying the alaninesolvent interaction.

We performed replica exchange Monte Carlo (REMC) simulations of the optimized sequence on a $200^{3}$ lattice with periodic boundary conditions. Each REMC simulation consisted of 64 replicas with a (reduced) temperature distribution around the transition temperature $(0.35 \leq$ $T^{*} \leq 0.45$ ), optimized by a feedback-optimization algorithm [21]. A replica exchange was attempted each 1000 $\mathrm{MC}$ cycles. After equilibrating for $1 \times 10^{10} \mathrm{MC}$ cycles per replica, we performed $5 \times 10^{10} \mathrm{MC}$ cycles for production. Employing the virtual-move parallel tempering method 22. ensured optimal use of the simulation data.

For a single polypeptide chain, corresponding to infinite dilution, we obtained the folding temperature from the heat capacity peak. Fig. 1 shows that this temperature is weakly dependent on the alanine hydrophobicity; at high $\epsilon_{A, w}$ the folded chain is slightly more stable, possibly because alanine side chains are more shielded 

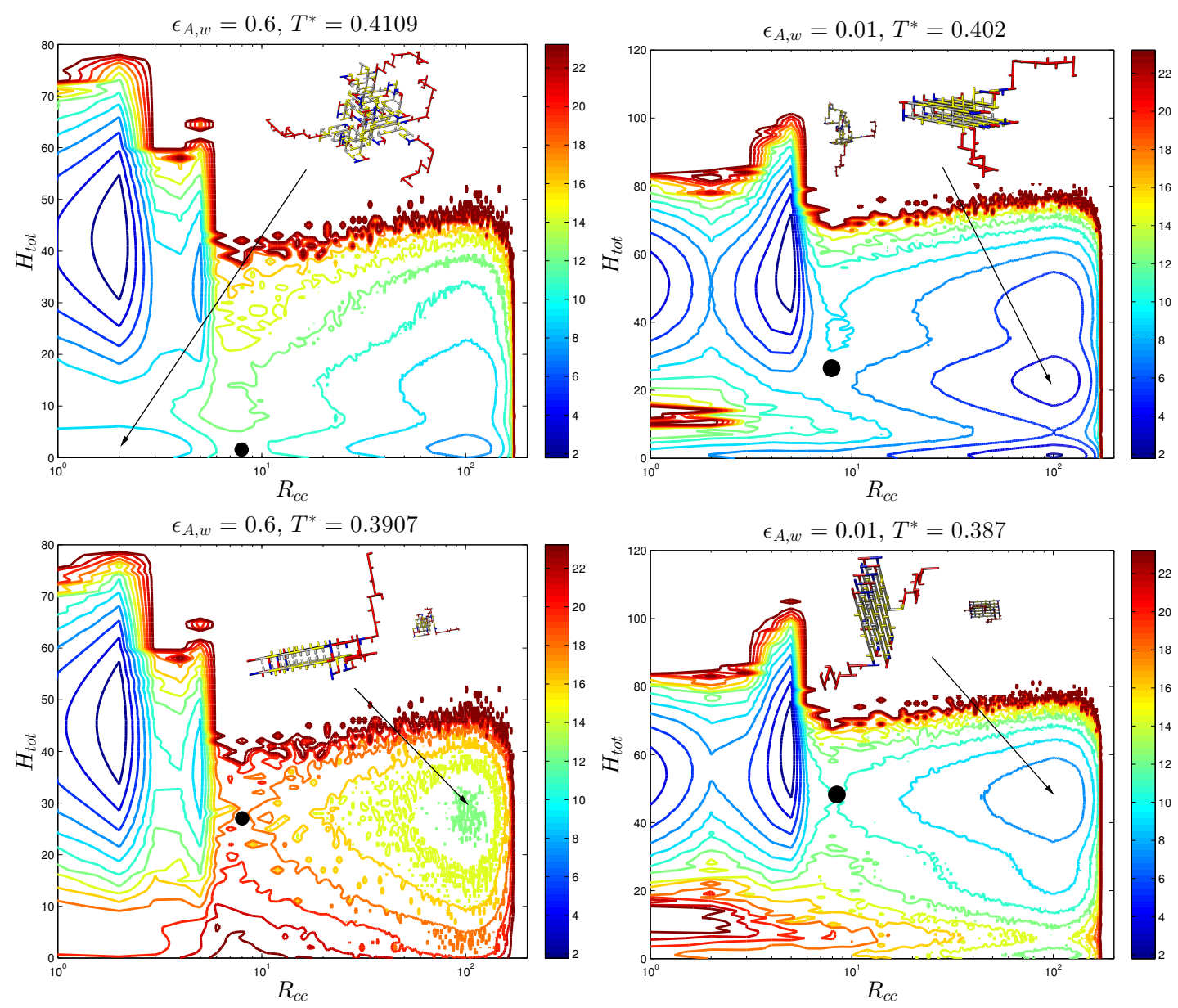

FIG. 2: (color online) Free energy landscape as a function of the silk-block center-to-center distance $R_{c c}$, and the total number of hydrogen bonds, $H_{t o t}$. Left column: $\epsilon_{A, w}=0.6, T^{*}=0.4109$ (top) and 0.3907 (bottom). Right column: $\epsilon_{A, w}=0.01$ at the temperature $T^{*}=0.402$ (top) and 0.387 (bottom). Solid circles denote saddle points.

from the water. In addition, with increasing hydrophobicity we observe more collapsed structures in the unfolded state.

Next, we performed REMC simulations of two polypeptides. Assuming a lattice space of $0.38 \mathrm{~nm}$ [17, this corresponds to a concentration of $7.57 \mu \mathrm{M}$. At high temperature the polypeptides are unfolded and mostly separated (see top left of Fig. 1). Upon decreasing temperature, a sharp peak in the heat capacity signals a transition to a self-assembled folded state, with a structure that depends on the alanine hydrophobicity $\epsilon_{A, w}$. For $\epsilon_{A, w}<0.2$, the two folded $\beta$-rolls align side-by-side. As in this regime the transition temperature remains almost constant, the formation of aligned state is not driven by the hydrophobicity of the $\beta$-roll surface, but instead by hydrogen bonds between the sides of two $\beta$-rolls. For $\epsilon_{A, w} \geq 0.2$, the two folded $\beta$-rolls are stacked on top of each other. In this regime the transition temperature increases monotonically with $\epsilon_{A, w}$ as the stacked state is stabilized by the hydrophobicity of the outside surface of the folded $\beta$-roll.
To shed light on the self-assembly mechanism of fibrilforming polypeptides, we plot in Fig. 2(left), for two temperatures, the free energy landscapes associated with the stacked state formation at $\epsilon_{A, w}=0.6$ as a function of the total number of hydrogen bonds, $H_{t o t}$, and the silk-block center-to-center distance $R_{c c}$. For both temperatures the global free energy minimum at $\left(R_{c c}=2, H_{t o t} \simeq 45\right)$ corresponds to the stacked state, and a local minimum at $\left(R_{c c} \simeq 5, H_{t o t} \simeq 35\right)$ represents the metastable aligned state. However, the mechanistic folding pathways differ significantly for the two temperatures. At $T^{*}=0.4109$, above the single polypeptide folding temperature, there exists (besides the minimum at $\left(R_{c c} \simeq 100, H_{t o t} \simeq 0\right)$ corresponding to isolated random coils) a second local minimum at $\left(R_{c c} \simeq 2, H_{t o t} \simeq 0\right.$ ), associated with a disordered aggregate of two polypeptides. The presence of this aggregated state causes the folding mechanism to pass through a saddle point around $\left(R_{c c} \simeq 8, H_{t o t} \simeq 1\right)$. This implies that above the single polypeptide folding temperature the self-assembly follows the pathway: unfolded state $\rightarrow$ random aggregation $\rightarrow$ stacked state. 
At $T^{*}=0.3907$, below the single polypeptide folding temperature, the free energy landscape changes significantly, and features a local minimum around $\left(R_{c c} \simeq\right.$ $100, H_{t o t} \simeq 30$ ), which corresponds to an intermediate state consisting of a separated folded and unfolded polypeptide. The channel connecting this state to the stacked state travels through a saddle point around $\left(R_{c c} \simeq 10, H_{t o t} \simeq 30\right)$, suggesting that the assembly is a self-templated process [23, in which a polypeptide folds on top of an already folded polypeptide. At very low temperatures the two polypeptides may fold independently before stacking together. However, we failed to observe such folding-docking process down to $T^{*}=0.35$, more than $15 \%$ below the transition temperature. Apparently, this is a very unlikely process, which explains why in experiments individual $\beta$-rolls are rarely observed, whereas they readily form on the growing end of an existing filament 9.

For an alanine hydrophobicity of $\epsilon_{A, w}=0.01$ typical free energy landscapes in Fig. 2(right) show that below the transition temperature, the global minimum is located at $\left(R_{c c} \simeq 5, H_{t o t} \simeq 60\right)$, corresponding to the aligned state (see Fig. 1). For $T^{*}=0.402$, just below the transition temperature, the assembly already occurs via the self-templated process, as indicated by the saddle point at $\left(R_{c c} \simeq 8, H_{t o t} \simeq 25\right)$ and the metastable intermediate state located at $\left(R_{c c} \simeq 100, H_{t o t} \simeq 25\right)$. The transition temperature is very close to the single polypeptide folding temperature (see Fig. 1), revealing that the binding between the folded polypeptides is weak. At $T^{*}=0.387$ one finds individual folded polypeptides in the solution, as indicated by a local minimum at $\left(R_{c c} \simeq 100, H_{t o t} \simeq 50\right)$. The saddle point at $\left(R_{c c} \simeq 10, H_{t o t} \simeq 50\right)$ suggests that the assembly then occurs via the folding-docking process. The local minimum at $\left(R_{c c} \simeq 1, H_{t o t} \simeq 50\right)$ corresponds to a metastable structure consisting of two interlocked folded $\beta$-sheets, which is probably an artifact of the model. In contrast to the strong hydrophobicity case, there is no third pathway via a random aggregate. We note that the high hydrophobicity case is probably a more realistic approximation to the experiments of Ref. 9].

To investigate the effect of polypeptide concentration, we performed REMC simulations at concentrations ranging from 1 to $60 \mu \mathrm{M}$, by changing the lattice size. Figure 3 shows the heat capacity for $\epsilon_{A, w}=0.6$ as a function of temperature. The decreasing height of the heat capacity peak with decreasing concentration indicates the self-assembly process becomes less cooperative at lower concentration. Still, the self-assembly transition temperature is always above the single polypeptide folding temperature, and the mechanism does not change. The inset in Fig. 3 shows the linear relationship between the reciprocal transition temperature and the logarithm of polypeptide concentration. We explain this behavior as follows. If assembly of two peptides yields an energy

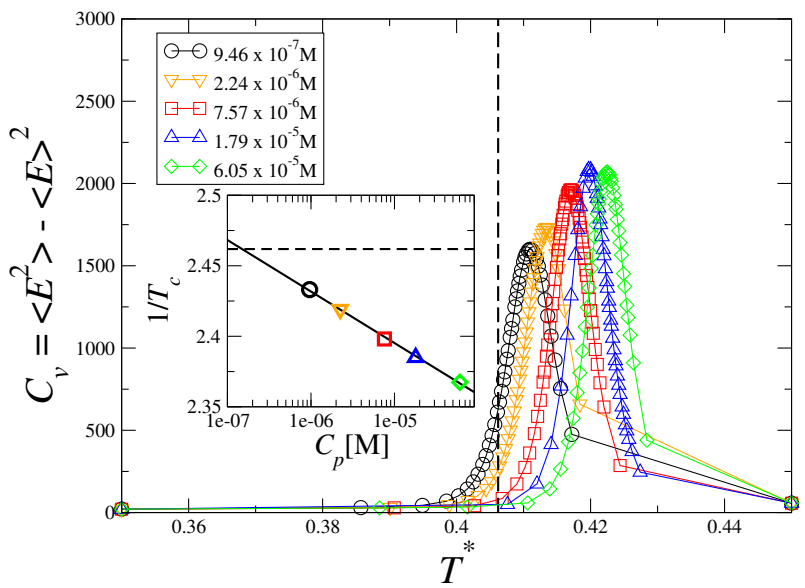

FIG. 3: (color online) Main: Heat capacity versus temperature for two polypeptides at various concentrations with $\epsilon_{A, w}=0.6$. Inset: The reciprocal transition temperature $T_{c}$ versus the logarithm of peptide concentration. In both plots the dashed line indicates the single polypeptide folding temperature.

gain $\Delta E<0$, then at the transition temperature $T_{c}$ the free energy difference between the isolated and assembled state is $\Delta F=\Delta E-T_{c} \Delta S=0$, where $\Delta S$ is the entropy lost in the assembly. Assuming ideal gas behavior of isolated peptides $\Delta S=k_{B} \ln C_{p}-\Delta S_{c}$, where $C_{p}$ is the polypeptide concentration. The first term is the translational entropy lost, while the second term is the conformational entropy lost $\left(\Delta S_{c}>0\right)$. It follows that $1 / T_{c}=\left(k_{B} / \Delta E\right) \ln C_{p}-\Delta S_{c} / \Delta E=a_{1} \ln C_{p}+a_{2}$, with $a_{1}<0$ and $a_{2}>0$, as we find in the simulations. We note that at very low concentration $\left(C_{p}<0.1 \mu M\right)$ the assembly will not influence the folding temperature anymore, as indicated by the dashed line in the inset. We speculate that below this concentration a folding-docking mechanism may indeed be present. For very high concentration $C_{p} \sim 1 / 8 R_{g}^{3}$, with the $R_{g}$ the radius of gyration, the unfolded chains are touching, and $T_{c}$ will also become independent of concentration.

In conclusion, we used a coarse-grained lattice model to systematically study the interplay between folding and assembly in the formation of hierarchically organized polypeptide fibrils. Despite the simplicity of the model, the results not only show a remarkably rich phase behaviour, but also, and most importantly, reveal a generic mechanism for protein fibril formation, depending on the relative strength of the intermolecular driving force for assembly (the hydrophobicity of the folded protein surface) with respect to the strength of the intramolecular driving force for protein folding (the hydrogen bonding). Coupling between these driving forces leads to an effect similar to allostery where a conformation change goes along with binding, and produces a templated-folding growth process at temperatures below the melting point. The model explains why experiments rarely detect the 
presence of individual $\beta$-rolls, but instead provide evidence for a templating mechanism for nucleation and growth 9 .

Our results show that the formation and growth of fibril structures can be tuned by varying the temperature and/or the hydrophobicity of specific residues, and are thus not only of fundamental interest, but may also provide guidance to fabricating novel bio-fibrils with high quality in experiments.

We thank Erik van Dijk of Vrije Universiteit Amsterdam for helpful discussions. We acknowledge financial support from the European Research Council through Advanced Grant 267254 (BioMate). This work is part of the research programme VICI 700.58.442, which is financed by the Netherlands Organization for Scientific Research (NWO). SA has been supported by a Veni grant on the project 'Understanding toxic protein oligomers through ensemble characteristics' from Netherlands Organization for Scientific Research (NWO).

* Electronic address: ran.ni@wur.nl

[1] C. M. Dobson, Trends Biochem. Sci. 24, 329 (1999).

[2] F. Chiti and C. M. Dobson, Annu. Rev. Biochem. 75, 333 (2006).

[3] C. Dobson, Nature (London) 426, 884 (2003).

[4] C. Haass and D. J. Selkoe, Nat. Rev. Mol. Cell Biol. 8, 101 (2007).

[5] T. Scheibel, R. Parthasarathy, G. Sawicki, X. M. Lin, H. Jaeger, and S. L. Lindquist, Proc. Natl. Acad. Sci. USA 100, 4527 (2003).

[6] K. J. Channon, G. L. Devlin, and C. E. MacPhee, J. Am.
Chem. Soc. 131, 12520 (2009).

[7] A. A. Martens, G. Portale, M. W. T. Werten, R. J. deVries, G. Eggingk, M. A. Cohen-Stuart, and F. A. deWolf, Macromolecules 42, 1002 (2009).

[8] M. Schor, A. A. Martens, F. A. de Wolf, M. A. CohenStuart, and P. G. Bolhuis, Soft Matter 5, 2658 (2009).

[9] H. B. Lennart, X. J. Beaudoux, J. M. Kleijn, F. A. de Wolf, and M. A. Cohen-Stuart, ACS Nano 6, 133 (2012).

[10] A. Irbäck, S. A. Jónsson, N. Linnemann, B. Linse, and S. Wallin, Phys. Rev. Lett. 110, 058101 (2013).

[11] S. Auer and D. Kashchiev, Phys. Rev. Lett. 104, 168105 (2010).

[12] J. E. Straub and D. Thirumalai, Annu. Rev. Phys. Chem. 62, 437 (2011).

[13] M. Schor and P. G. Bolhuis, Phys. Chem. Chem. Phys. 13, 10457 (2011).

[14] H. D. Nguyen and C. K. Hall, Journal of the American Chemical Society 128, 1890 (2006).

[15] V. A. Wagoner, M. Cheon, I. Chang, and C. K. Hall, Journal of Molecular Biology 416, 598 (2012).

[16] E. M. Phelps and C. K. Hall, Proteins: Structure, Function, and Bioinformatics 80, 1582 (2012).

[17] S. Abeln and D. Frenkel, Biophysical Journal 100, 693 (2011).

[18] S. Abeln and D. Frenkel, submitted (2013).

[19] See Supplimentary Material.

[20] S. Abeln and D. Frenkel, PLoS computational biology 4, e1000241 (2008).

[21] H. G. Katzgraber, S. Trebst, D. A. Huse, and M. Troyer, Journal of Statistical Mechanics: Theory and Experiment 2006, P03018 (2006).

[22] I. Coluzza and D. Frenkel, ChemPhysChem 6, 1779 (2005).

[23] S. Auer, C. M. Dobson, M. Vendruscolo, and A. Maritan, Phys. Rev. Lett. 101, 258101 (2008). 